\title{
Clinical study of first-line endocrine therapy for type ER+/PR+ and ER+/PR- advanced breast cancer
}

\author{
Mengwei Zhang, Min Yan, Huimin Lv, Limin Niu, Huiai Zeng \\ Department of Breast disease, Henan Breast Cancer Center, The Affiliated Cancer Hospital of Zhengzhou University \& Henan Cancer Hospital, \\ Zhengzhou, China \\ Contributions: (I) Conception and design: M Zhang; (II) Administrative support: M Yan; (III) Provision of study materials or patients: M Zhang, M \\ Yan, H Lv, L Niu, H Zeng; (IV) Collection and assembly of data: M Zhang; (V) Data analysis and interpretation: M Zhang; (VI) Manu-script writing: \\ All authors; (VII) Final approval of manuscript: All authors. \\ Correspondence to: Min Yan. The Affiliated Cancer Hospital of Zhengzhou University \& Henan Cancer Hospital, No.127, Dongming Road, Zhengzhou, \\ China. Email: ym200678@126.com.
}

\begin{abstract}
Background: This study was designed to evaluate the relationship between estrogen receptor (ER) and progesterone receptor (PR) expression status and clinical outcomes in advanced breast cancer patients undergoing first-line endocrine therapy.

Methods: Data from 225 advanced breast cancer patients admitted to Henan Cancer Hospital from February 2010 to October 2019 were collected and compared using Chi-squared tests, with Cox regression models being used to identify relevant prognostic factors in these patients.

Results: PR+ and PR- patients had significantly different median progression-free survival (PFS) times of 25 months (95\% CI: 13.50-36.50) and 7 months (95\% CI: 4.03-9.97), respectively $(\mathrm{P}<0.001)$. Clinical benefit rates (CBR) were also significantly different between these 2 groups $(80.9 \%$ and $55.6 \%$, respectively; $\mathrm{P}<0.001)$. A subgroup analysis of $\mathrm{PR}+$ and $\mathrm{PR}$ - patients that underwent aromatase inhibitor (AI) treatment revealed a median PFS of 25.0 months (95\% CI: 14.28-35.72) and 7 months (95\% CI: 4.18-9.82), respectively $(\mathrm{P}<0.001)$, and $\mathrm{CBR}$ values of $81.3 \%$ and $54.5 \%$, respectively $(\mathrm{P}<0.001)$. In addition, for both the whole cohort and the AI subgroup, the total survival of patients with $\mathrm{ER}+\mathrm{PR}+$ breast cancer was longer than that of patients with $\mathrm{ER}+\mathrm{PR}$ - breast cancer, and the difference was statistically significant $(\mathrm{P}<0.001)$.

Conclusions: ER+/PR+ advanced breast cancer patients had a better prognosis than ER+/PR- advanced breast cancer patients undergoing first-line endocrine therapy. In addition, we found that PR status was an independent predictor of first-line endocrine therapy responses in hormone receptor-positive HER2 negative patients.
\end{abstract}

Keywords: Estrogen receptor (ER)+/progesterone receptor (PR)+; ER+/PR-; first-line endocrine therapy

Submitted Sep 29, 2020. Accepted for publication Jan 17, 2021.

doi: 10.21037/apm-20-2180

View this article at: http://dx.doi.org/10.21037/apm-20-2180

\section{Introduction}

Hormone receptor (HR)-positive advanced breast cancer generally progresses slowly and has a relatively good prognosis, and most tumors of this type retain their biological characteristics. Endocrine therapy is generally the clinical approach of choice in these patients, as their short-term prognosis is good. Estrogen receptor (ER)+/ progesterone receptor (PR)- breast cancer was originally identified by Perou et al. (1), and was classified as luminal B-type breast cancer at the genetic level, consistent with the findings produced by Chin et al. (2). ER+/PR- breast cancers generally exhibit increased genetic instability, enhanced tumor cell proliferation, and reduced patient survival relative to $\mathrm{PR}+$ breast cancers. To date, several studies have evaluated the relationship between PR status and treatment outcomes in advanced $\mathrm{HR}+$ breast cancer. As such, in the present study, we analyzed retrospective clinical 
Table 1 Characteristics of the study patients.

\begin{tabular}{|c|c|c|}
\hline Clinicopathological parameters & $\mathrm{ER}+/ \mathrm{PR}+$ & $\mathrm{ER}+/ \mathrm{PR}-$ \\
\hline Median age (years) & 50 & 50 \\
\hline \multicolumn{3}{|c|}{ Menstrual status (menopause), n (\%) } \\
\hline Yes & $87(53.7)$ & $33(52.3)$ \\
\hline No & $75(46.2)$ & $30(47.6)$ \\
\hline \multicolumn{3}{|c|}{ Disease-free survival time (DFS), n (\%) } \\
\hline IV phase of first visit & $13(8)$ & $4(6.3)$ \\
\hline$\leq 12$ months & $7(4.2)$ & $2(3.0)$ \\
\hline$>12$ months & $142(87.8)$ & $57(90.7)$ \\
\hline \multicolumn{3}{|l|}{ Metastasis site, n (\%) } \\
\hline Lymph node or soft tissue & $83(51.2)$ & $32(50.7)$ \\
\hline Liver & $53(32.7)$ & $32(50.7)$ \\
\hline Lung & $69(42.5)$ & $27(42.8)$ \\
\hline Bone & $112(69.1)$ & $44(69.8)$ \\
\hline \multicolumn{3}{|l|}{ Number of transfer sites, n (\%) } \\
\hline 2 or less & $103(63.4)$ & $3(53.9)$ \\
\hline More than 2 & $59(36.6)$ & $60(46.1)$ \\
\hline \multicolumn{3}{|l|}{ Endocrine therapy, n (\%) } \\
\hline Aromatase inhibitor & $134(82.8)$ & $55(87.3)$ \\
\hline Fulvestrant group & $28(17.2)$ & $8(12.7)$ \\
\hline
\end{tabular}

data from 225 advanced breast cancer patients undergoing treatment with either an aromatase inhibitor (AI) or fulvestrant. We present the following article in accordance with the REMARK reporting checklist (available at http:// dx.doi.org/10.21037/apm-20-2180).

\section{Methods}

\section{Patients}

Between January 2010 and October 2019, 225 breast cancer patients with local or distantly metastasized HR+/HER2status underwent first-line treatment with AI $(n=189)$ or fulvestrant ( $\mathrm{n}=36)$ at the Henan Cancer Hospital. Complete clinical data were available for all of these patients. Patient inclusion criteria for the present study were as follows: (I) patients had pathologically-diagnosed breast cancer that had undergone local or distant metastasis as demonstrated through 2 imaging evaluations, without any symptoms associated with visceral metastases; (II) clinical data, including treatment and follow-up data, were complete and all patients were able to complete treatment and follow- up; (III) ER and PR status was detected, with PR+ being determined based on $>20 \%$ PR+ tumor cells (3); (IV) patients did not undergo any additional chemotherapy or endocrine therapy within the first 4 weeks of treatment; and (V) patient blood, heart, lung, liver, and kidney functional analyses were essentially normal (Eastern Cooperative Oncology Group score $0-3$, Table 1). The study was conducted in accordance with the Declaration of Helsinki (as revised in 2013). The study was approved by institutional ethics board of Henan Cancer Hospital (No.: 2019102821) and informed consent was taken from all individual participants.

\section{Treatment}

Patients in the AI treatment group received anastrozole $(1 \mathrm{mg})$, letrozole $(2.5 \mathrm{mg})$, or exemestane $(25 \mathrm{mg})$ once per day for 4 consecutive weeks ( 1 cycle). Patients in the fulvestrant treatment group received an intramuscular injection of fulvestrant $(500 \mathrm{mg})$ on days $0,14,28$, and every 28 days thereafter ( 1 cycle). In addition, premenopausal patients were treated with a subcutaneous injection of goserelin $(3.6 \mathrm{mg})$ once every 28 days.

\section{Evaluation criteria}

According to the RECIST 1.1 criteria, treatment-associated curative effects were classified into complete remission (CR), partial remission (PR), stable disease (SD), and progressive disease (PD) subtypes. Clinical benefit rate (CBR) was defined based on the number of patients that achieved CR, $\mathrm{PR}$, or SD for $>6$ months. Progression-free survival (PFS) was defined as the time between treatment initiation and progression or death due to any cause. Overall survival (OS) was defined as the time between breast cancer diagnosis and death or most recent follow-up (March 31, 2020). First-line endocrine therapy referred to endocrine therapy as the firstline rescue therapy.

\section{Follow-up}

Patient follow-up was conducted through outpatient visits, inpatient visits, or by telephone. Follow-up analyses were used to evaluate treatment, disease progression, and duration of clinical benefit. Every 1-2 cycles, target lesions underwent evaluation via spiral computed tomography (CT) or magnetic resonance imaging (MRI). All followup included in the present study was conducted on or before March 31, 2020, and the median follow-up time was 


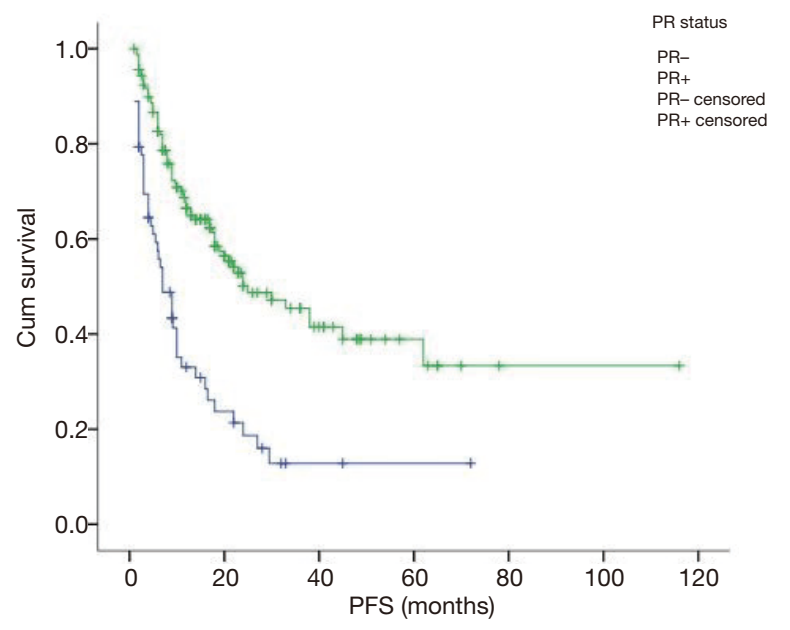

Figure 1 The progression-free survival (PFS) of progesterone receptor $(\mathrm{PR})+$ and $\mathrm{PR}$ - advanced breast cancer patients undergoing first-line endocrine therapy.

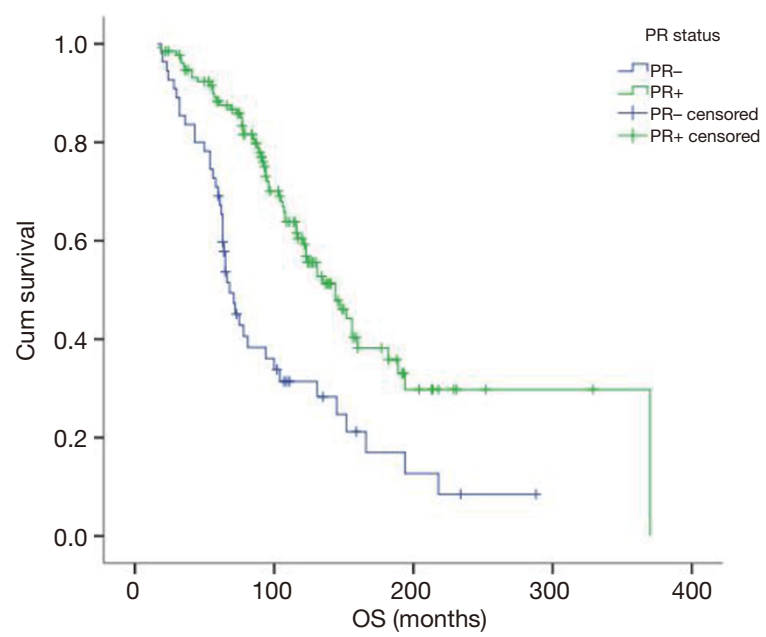

Figure 2 The overall survival (OS) of progesterone receptor (PR)+ and $\mathrm{PR}$ - advanced breast cancer patients undergoing first-line endocrine therapy.

18 months.

\section{Statistical analysis}

SPSS v18.0 was used for all statistical analyses. Survival analyses were conducted via the Kaplan-Meier method. Single-factor analyses were conducted via the log-rank test, while Cox proportional risk models were used for multivariate analyses. $\mathrm{P}<0.05$ was the significance threshold.

\section{Results \\ Clinical efficacy and tolerability}

The median PFS of patients in the PR+ and PR- groups was 25 months (95\% CI: 13.50-36.50) and 7 months (95\% CI: 4.03-9.97), respectively, with a significant difference in survival being observed between these groups $(\mathrm{P}<0.001$, Figure 1). CBR was also significantly different between these 2 groups $(80.9 \%$ vs. $55.6 \%$; $\mathrm{P}<0.001)$, as was OS [135.5 months (95\% CI: 114.90-156.11) vs. 71 months (95\% CI: 60.27-81.73), $\mathrm{P}<0.001$, Figure 2]. The median $\mathrm{PFS}$ of $\mathrm{PR}+$ and $\mathrm{PR}-$ patients in the AI treatment subgroup receiving first-line endocrine therapy was 25.0 months (95\% CI: $14.28-35.72)$ and 7 months (95\% CI: 4.18-9.82) respectively, while CBR values in these 2 groups were $81.3 \%$ and $54.5 \%$, respectively, with significant differences between groups in both cases $(\mathrm{P}<0.001$; Table 2). The OS in these 2 subgroups also differed significantly, from 144 months (95\% CI: 118.26-169.74) in the PR+ group, to 68 months (95\% CI: 58.00-78) in the $\mathrm{PR}-$ group $(\mathrm{P}<0.001)$. No significant adverse events occurred in these patients.

We next compared differences in PFS and OS in these patients as a function of factors including age, PR status, endocrine therapy type auxiliary stage, disease-free survival time (DFS), number of metastasis sites, presence or absence of liver metastases, and the presence or absence of lung metastases. We found that the median PFS of PR+ and PR-patients was 25.0 months and 7.0 months, respectively, while the CBR was $80.9 \%$ and $55.6 \%$, respectively, with significant differences between groups $(\mathrm{P}<0.001)$. Patients with and without liver metastases had a median PFS of 50 months and 102 months, respectively, and a median OS of 89 months and 152 months, respectively, with significant differences between these groups $(\mathrm{P}<0.001)$.

\section{Prognostic analyses}

We next conducted a Cox regression analysis to identify factors associated with first-line endocrine therapy outcomes in AI subgroup patients. The following parameters were incorporated into this analysis: age at the time of firstline endocrine therapy ( $<50 v s . \geq 50$ years), hormone receptor status (PR+vs. PR-), whether endocrine therapy was accepted during the adjuvant stage (yes $v s$. no), DFS ( $<2 v s . \geq 2$ years), number of metastasis sites ( $\leq 2 v s .>2$ ), and whether visceral metastases were present (yes $v s$. no). The results of this analysis revealed that both PR status and liver 
Table 2 Single-factor and multiple factor analyses of progression-free survival (PFS) and overall survival (OS) in patients undergoing first-line endocrine therapy

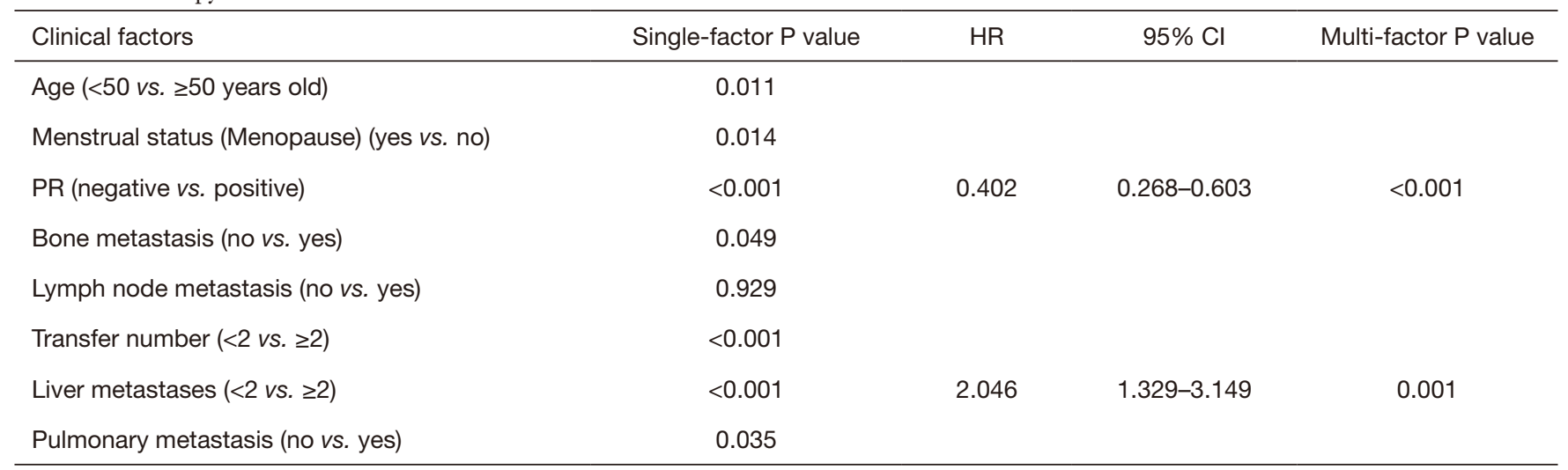

metastasis were independent predictors of median PFS and OS in first-line endocrine therapy patients (Table 2).

\section{Discussion}

As relatively stably expressed proteins, ER and PR represent effective functional targets on breast cancer cells when they are present. Endocrine therapy can disrupt estrogen synthesis or signaling, thus interfering with the ability of this hormone to drive tumor cell proliferation. Advances in endocrine therapy have led to its use as a primary treatment for patients with metastatic breast cancer along with surgery, chemotherapy, and radiotherapy, significantly extending median patient survival (18-24 months) (4). At present, National Comprehensive Cancer Network (NCCN) guidelines recommend that patients with $\mathrm{HR}+$ / HER2 - breast cancer with slowly progressive disease and without symptomatic visceral metastases should preferably undergo endocrine therapy as first-line treatment $(5,6)$.

ER and PR expression can predict tumor sensitivity to endocrine therapy. In recurrent tumors that are ER+ and $\mathrm{PR}+$, endocrine therapy can achieve curative efficacy in $50-75 \%$ of cases. Cure rates for ER- and PR- tumors are $<10 \%$. The biological, prognostic, and predictive importance of the assessment of ER expression is wellestablished in breast cancer, whereas the added value of PR expression status remains controversial. Some studies suggest that $\mathrm{PR}$ - tumors are more likely to be aggressive than $\mathrm{PR}+$ tumors in patients with ER+/HER2- breast cancer, and PR status determines tumor responsiveness to endocrine therapy in early breast cancer, such that PRtumors exhibit reduced tamoxifen sensitivity (7). AIs have been shown to exhibit superior efficacy when used for endocrine therapy, reducing the risk of recurrence by $52 \%$ relative to tamoxifen (8). In this study, we observed a CBR of $81.3 \%$ and $54.5 \%$ in $\mathrm{PR}+$ and $\mathrm{PR}$ - patients undergoing AI-based first-line endocrine therapy, respectively, suggesting that $\mathrm{PR}$ expression is associated with the efficacy of AI therapy in ER+ metastatic breast cancer patients, such that PR- patients are significantly less sensitive to AI therapy. Our results are consistent with studies of early breast cancer. Fulvestrant exhibited better therapeutic efficacy in this type of breast cancer and remains an effective treatment option in the context of AI resistance $(9,10)$. In this study, there were no significant differences in the number of cases of PR- in the fulvestrant group (8/36). This result may be related to the small number of cases in the PR- fulvestrant group.

In early-stage breast cancer patients, $\mathrm{ER}+/ \mathrm{PR}+$ status is associated with lower recurrence and a better prognosis than ER+/PR- status $(11,12)$. In this study, we conducted a retrospective analysis of $225 \mathrm{HR}+$ metastatic breast cancer patients that had been treated with $\mathrm{AI}$ or fulvestrant. This overall patient cohort had a median survival of 18 months, with the ER+/PR+ and ER+/PR- groups having significantly different median PFS values of 25 and 7 months, respectively. Similarly, OS differed significantly between these groups. The OS of PR+ breast cancer patients has previously been shown to be longer than that of PR-patients (13), consistent with our results. In a multivariate analysis, we found that both PR status and liver metastasis were independent predictors of OS and PFS, suggesting that the characteristics of advanced HR+/HER2breast cancer are consistent with those of early-stage breast 
cancer. ER+/PR- patients exhibited a poorer prognosis and therapeutic responsiveness, indicating that these patients require more attention in clinical practice. Particularly for patients with liver metastases, our data additionally highlights the importance of optimizing endocrine therapy and chemotherapy timing and treatment in order to achieve longer patient survival.

In our study, we observed that PR status can affect PFS and OS associated with first-line endocrine therapy in patients with advanced ER+/HER2- breast cancer. Whether intensive chemotherapy and subsequent endocrine therapy in ER+/PR- patients is beneficial, however, still requires confirmation through large-scale prospective clinical studies. Furthermore, with the advent of CDK4/6 inhibitors such as palbociclib, future prospective clinical trials on the relationship between PR status and OS/PFS following treatment with CDK4/6 inhibitors and AI or fulvestrant are needed.

\section{Acknowledgments}

The authors would like to thank the previous researchers and scientists for their studies. Meanwhile, we were very grateful for the help provided by Henan cancer hospital and the contribution of all patients' information included in this study.

Funding: None.

\section{Footnote}

Reporting Checklist: The authors have completed the REMARK reporting checklist. Available at http://dx.doi. org/10.21037/apm-20-2180

Data Sharing Statement: Available at http://dx.doi. org/10.21037/apm-20-2180

Conflicts of Interest: All authors have completed the ICMJE uniform disclosure form (available at http://dx.doi. org/10.21037/apm-20-2180). The authors have no conflicts of interest to declare.

Ethical Statement: The authors are accountable for all aspects of the work in ensuring that questions related to the accuracy or integrity of any part of the work are appropriately investigated and resolved. The study was conducted in accordance with the Declaration of Helsinki (as revised in 2013). The study was approved by institutional ethics board of Henan Cancer Hospital (No.: 2019102821) and informed consent was taken from all individual participants.

Open Access Statement: This is an Open Access article distributed in accordance with the Creative Commons Attribution-NonCommercial-NoDerivs 4.0 International License (CC BY-NC-ND 4.0), which permits the noncommercial replication and distribution of the article with the strict proviso that no changes or edits are made and the original work is properly cited (including links to both the formal publication through the relevant DOI and the license). See: https://creativecommons.org/licenses/by-nc-nd/4.0/.

\section{References}

1. Perou CM, Sørlie T, Eisen MB, et al. Molecular portraits of human breast tumours. Nature 2000;406:747-52.

2. Chin K, DeVries S, Fridlyand J, et al. Genomic and transcriptional aberrations linked to breast cancer pathophysiologies. Cancer Cell 2006;10:529-41.

3. Prat A, Cheang MC, Martín M, et al. Prognostic significance of progesterone receptor-positive tumor cells within immunohistochemically defined luminal A breast cancer. J Clin Oncol 2013;31:203-9.

4. Er O, Frye DK, Kau SW, et al. Clinical course of breast cancer patients with metastases limited to the liver treated with chemotherapy. Cancer J 2008;14:62-8.

5. Jiang L, Jiang Z. Investigation and research on the current situation of diagnosis and treatment of advanced breast cancer in china. Cancer Res Clinic 2013;25:481-4.

6. Jiang Z, Xu B, Shao Z. Basic principles of chemotherapy for relapsed and metastatic breast cancer. Chin Med J 2011;91:73-5.

7. Bulut N, Altundag K. Does estrogen receptor determination affect prognosis in early stage breast cancers? Int J Clin Exp Med 2015;8:21454-9.

8. Yu KD, Liu GY, Di GH, et al. Progesterone receptor status provides predictive value for adjuvant endocrine therapy in older estrogen receptor-positive breast cancer patients. Breast 2007;16:307-15.

9. Cui X, Schiff R, Arpino G, et al. Biology of progesterone receptor loss in breast cancer and its implications for endocrine therapy. J Clin Oncol 2005;23:7721-35.

10. Zhang $\mathrm{Y}, \mathrm{Su} \mathrm{H}$, Rahimi M, et al. EGFRvIII-induced estrogen-independence, tamoxifen-resistance phenotype correlates with PgR expression and modulation of apoptotic molecules in breast cancer. Int J Cancer 
2009;125:2021-8.

11. Aapro MS, Forbes JF. Three years' follow-up from the ATAC trial is sufficient to change clinical practice: a debate. Breast Cancer Res Treat 2003;80 Suppl 1:S3-11; discussion $\mathrm{S} 13-8$.

12. Zhang C, Liu Z, Ma Q. Clinical study of estrogen progesterone receptor positive and estrogen receptor positive progesterone receptor negative breast cancer.
Chin J Oncol 2017;9:149-53.

13. Gao J, Tang W, Xi G. Er+/pr- type breast cancer and type $\mathrm{er}+\mathrm{pr}+$ breast cancer clinicopathological features and prognosis analysis. Chin J Clin Phy 2016;10:603-6.

(English Language Editor: C. Betlazar-Maseh)

Cite this article as: Zhang M, Yan M, Lv H, Niu L, Zeng $\mathrm{H}$. Clinical study of first-line endocrine therapy for type ER+/ $\mathrm{PR}+$ and ER+/PR- advanced breast cancer. Ann Palliat Med 2021;10(1):238-243. doi: 10.21037/apm-20-2180 